\title{
LA GESTIÓN TERAPEÚTICA DE LA CULTURA: UNA NUEVA FÓRMULA DE DISCIPLINA SOCIAL
}

\author{
Lina Gavira Álvarez \\ Universidad de Sevilla
}

\section{Resumen.}

La crisis y la incertidumbre actual está derivando en una progresiva "terapeutalización de la cultura", que supone un nuevo modelo de control social desde las emociones, basado en trasladar el expertismo psicologísta al campo de la acción social, para promover el "estar bien", reformulando las bases del "bienestar". Esta estrategia erosiona los derechos de ciudadanía, al propiciar una identidad pasiva, como pacientes. Las manifestaciones culturales o el arte quedan bajo sospecha, cuando usan códigos que no participan de lo establecido por las élites como "adecuado". Así desde la política se hace una apuesta generalizada por la "instrucción emocional", mientras se invisibilizan las dimensiones culturales y materiales que permiten afrontar los problemas desde una lógica activa de ciudadanía que apueste por una calidad de vida más humana. En este contexto, los profesionales de la cultura como mediadores tienen un papel importante ante la encrucijada de promover proyectos en formatos que propicien una cultura transformadora, o bien ser un agente más de la reproducción del malestar y la terapia cultural paliativa ${ }^{1}$.

Palabras clave: Cultura, salud, control social, bienestar, terapeutalización, gestiónecorresponsable, glocalización.

1. La parte central de esta aportación ha sido presentada como ponencia en el Congreso Internacional de Gestión Cultural celebrado en Almería. 


\begin{abstract}
.
The crisis and the current uncertainty is leading to a progressive "therapeutic culture", which is a new model of social control of emotions, based on moving the psychologistic expertism on the field of social action to promote "be well" by reformulating the basis of "well-being". This strategy undermines citizenship rights by fostering a passive identity, as patients. Cultural events and art are under suspicion when using codes not established by the elites as "adequate." There is a political bet on "emotional statement" while they obscure the cultural and material dimensions that can face problems from a logical active citizenship with a commitment for a more human quality of life. In this context, cultural professionals as mediators have an important role at the crossroads of formats to promote projects that foster a culture capable of transforming or be one more agent reproducing discomfort and palliative cultural therapy ${ }^{2}$.
\end{abstract}

Keywords: Culture, health care, social control, well-being, therapeutic, eco-responsible management, glocalisation.

\title{
1. INTRODUCCIÓN
}

En la sociedad informacional (Castells, 1985) se considera que el conocimiento es uno de los pilares fundamentales para la obtener poder, riqueza y buena calidad de vida, lo que afecta directamente a los campos del bienestar, la salud y la cultura. Uno de los problemas que se plantea en este ámbito en la actualidad se refiere al papel que tienen los mediadores o profesionales de las instituciones culturales en el proceso de reproducción cultural, ámbito tratado ampliamente por Bourdieu y Passeron y sus seguidores desde una perspectiva materialista y actualizado en el análisis crítico realizado por Bolstanki y Chiapello (2002) en el nuevo espíritu del capitalismo señalando cómo penetra la ideología entre los grupos técnicos mediadores en el ejercicio de las políticas.

En el caso de los técnicos orientados a la gestión de la cultura entendida como "cultura artística", "cultura popular" o "industria cultural" plantea un problema de análisis interesante, en la medida de que en pocas ocasiones tienen presente el papel que pueden tener como agentes mediadores orientados a la reproducción o la transformación de actitudes ante la vida de las poblaciones a las que dirigen sus programas e intervenciones. En la sociedad informacional una de las variables fundamentales para no quedar desafiliado (Castel, 2004) consiste en tener la capacidad de discernir sobre la legitimidad tanto de los conocimientos como de las pautas e informaciones que reciben desde los diferentes campos de conocimiento sobre los que operan, a partir de fuentes de

2. The central part of this contribution has been presented as a paper at the International Congress of Cultural Management held in Almería. 
información cada vez más amplias y diversas, en las que resulta muy complejo identificar qué fines los inspiran, ya que la ciencia, como conocimiento legitimado, se ve atravesada, como cualquier otra institución, por las luchas de poder e intereses que afectan a la vida social.

En la etapa actual de capitalismo informacional esto resulta más preocupante que en la sociedad industrial, debido a la progresiva globalización socioeconómica y cultural, tanto como a las estrategias de desregulación que propicia en nuestras sociedades, lo que suele conducir a una mayor falta de control democrático tanto del acceso como del uso del conocimiento.

Es importante subrayar, que siempre estamos construyendo mapas cognitivos de la realidad sobre la que actuamos y que las herramientas que utilizamos para construirlos vienen mediadas por los sistemas de control capitalísticos (Guattari y Rolnik, 2006) que afectan a las definiciones de los términos tratados y a los enfoques que se les aplica en cada etapa histórica. Por ello conviene, en aras de la claridad expositiva, delimitar las relaciones que se establecen entre gestión cultural y salud social, así como definir previamente qué sentido alcanzan estos términos, por más familiares que puedan resultar para la mayoría de los lectores.

\section{RELACIONES ENTRE SALUD Y CULTURA EN EL CONTEXTO DE LA SOCIEDAD INFORMACIONAL}

La definición de salud consensuada por la Organización Mundial de la Salud (OMS) de Naciones Unidas entiende por salud no la falta de enfermedad, sino el estado de completo bienestar físico, mental y social, y no solamente la ausencia de afecciones o enfermedades: hay personas enfermas con más salud que otras personas aparentemente sanas, si nos atenemos a esta definición, igual que hay sociedades sanas a pesar de su apariencia de malestar derivado de prácticas austeras de consumo equilibradas con el medio en el que se localizan. De hecho, la primera variable que la OMS ha identificado que guarda una relación directa con la salud es la educación, no la medicación. Esto otorga a la cultura un papel central como ámbito por antonomasia de la educación formal e informal y una enorme relevancia como vía para mantener el derecho a la salud biofísica que junto a la cultura nos constituye como especie.

El modelo de organización social y el tipo de relaciones sociales que propicia a través las instituciones y los procesos que desarrollan, va a ser fundamental para promover la salud y el bienestar o, por el contrario, favorecer el malestar. En la etapa de glocalización (Roberston, 1993; Beck, 1998; Gavira, 1995, 2002) y crisis que vivimos, se constata un creciente malestar en la cultura o corrosión del carácter (Sennett, 2000) que no es ajeno a las formas de organización dominantes.

Este malestar en la cultura informacional se concreta en una percepción creciente 
de la incertidumbre ante la progresiva velocidad de los cambios que estamos viviendo, que afecta a la concepción del tiempo y el espacio, haciendo de la instantaneidad de los procesos un valor, que, en muchos casos, lleva a que nuestros cuerpos y su reloj biológico no puedan soportar el tiempo que imponen las organizaciones.

De otro lado, la progresiva globalización de la comunicación, la flexibilidad y movilidad de los dispositivos, también tienden una segunda trampa a nuestra percepción, haciéndonos creer que si bien parece que el mundo se ha hecho más cercano, en cambio, los procesos en los que creemos que podemos intervenir, son mucho más complejos y más fuera de control. Esto conduce a la creencia de que intervenir en el espacio mundo de forma organizada es prácticamente imposible, lo que lleva a que una gran parte de la ciudadanía renuncie al ejercicio activo de controlar la propia vida, ya que ahora creen ajeno a sus posibilidades de acción y en consecuencia, también abandonan el ejercicio de gobernanza desde lo cotidiano, renunciando a la participación efectiva en las organizaciones con las que conviven día a día, perdiendo también la fe en la capacidad de intervención en el espacio glocal, sentido cada vez más lejano y fuera de control.

No es ajeno a todo ello, la estrategia comunicativa de los medios de promoción del miedo al otro, inyectado en nuestra realidad cotidiana como estrategia de disciplina social a través de noticias sobre desastres ecológicos, económicos, terrorismo, violencia urbana y doméstica... lo que aumenta la incertidumbre, en paralelo a la creencia de que el mundo, nuestro mundo cotidiano, tanto personal como laboral o social se convierte en un "campo de batalla" (Barman, 2001), donde los fuertes son los que ganan. Esto es muy funcional para los grupos de poder que alimentan estas estrategias de atomización e individualización social desde la apuesta por un modelo de competitividad, que si bien se plantea en muchos casos con un discurso meritocrático, se contrapone con la experiencia vivida de desregulación y corrupción, haciendo que se termine interiorizando la competitividad como un "sálvese quien pueda".

La mala salud aumenta si a lo anterior se añade la crisis e incertidumbre económicofinanciera-política con sus lacras de paro, pobreza, movilidad de poblaciones,... que retroalimenta una crisis social sin precedentes como consecuencia de los procesos de fragmentación e individualización, que está dejando a las clases populares sin referente de organización colectiva para defender sus derechos. A esta mala salud derivada de la nueva división del trabajo, el cambio en las formas de organización, que hace posible que los trabajadores pobres y muy cualificados debido a la precariedad aumenten en Europa a ritmo acelerado, se añaden los procesos de cambio de la vida personal, familiar y doméstica, propiciados tanto por la nueva situación económica, como por las posibilidades de adquirir identidades múltiples a través de las redes virtuales, haciendo más débiles los límites que separan el mundo de lo privado y de lo público. Por otra parte, la frivolización y espectacularización de las relaciones de vecindad, trabajo o 
familia hacen que fallen muchos de los mecanismos de solidaridad y apoyo mutuo que tradicionalmente han constituido la base de lo social, sustituyéndolas en el mejor de los casos por vínculos débiles construidos en las redes sociales virtuales, ante la mirada oculta de aquellos que los utilizan como negocio, haciendo que paradójicamente se puedan tener miles de "amigos" virtuales y un fuerte sentimiento de soledad y falta de apoyo real y cotidiano.

A lo anterior, se suma la crisis ideológica y de valores, que lleva a la búsqueda de nuevas creencias que les sirvan para orientarse en el mundo, una vez ampliados los marcos de referencia más allá del universo local, lo que provoca miedo y desmovilización, pero no evita el pánico ni que muchas de las iniciativas que surgen como reacción a este estado de cosas, sean temporales y fragmentarias. En este contexto la gente busca como nunca en la modernidad, espacios de protección de ayuda mutua y apoyo emocional, que dependiendo de las culturas más o menos familistas o de clan -por ejemplo, los países anglosajones no cuentan con la red familiar que cuentan los países mediterráneos- se obtiene con terapeutas profesionales o en pequeños grupos terapéuticos o vinculados a nuevos consumos de ocio, como fútbol, los gimnasios, los grupos de aficiones relacionadas con la New Age o grupos de inspiración religiosa que asumen en su formas de interactuar criterios terapéuticos.

\section{LA CONSTRUCCIÓN DE LA CULTURA TERAPÉUTICA, EMOCIONALISMO VERBAL Y CONTROL SOCIAL DE LA GESTIÓN DE LAS EMOCIONES.}

En este contexto, el discurso terapéutico experto va penetrando silenciosamente aprovechando la incertidumbre, colonizando el imaginario colectivo, subrayando la situación de vulnerabilidad de las poblaciones, incorporándose a nuestra cultura en todos los ámbitos de la vida. Fran Furedi (2004) ha analizado este fenómeno en el mundo anglosajón. Resulta impresionante, cuando se analiza sistemáticamente, comprobar cómo se usa el miedo de la gente para promocionar la vulnerabilidad y, de otro lado, poder observar sobre qué términos y espacios opera esa estrategia. Así este autor pone de manifiesto como el uso creciente de la jerga terapéutica en los medios no sólo tiene un interés lingüístico, subrayando cómo las formas cambiantes del idioma transmiten nuevas actitudes y expectativas culturales que orientan la acción colectiva.

Parece como si se estuviera dando un cambio de polaridad en las formas de decir y hacer, pasando de un materialismo extremo que ha olvidado la complejidad del ser humano a un emocionalismo que oculta las bases materiales y sociales de la existencia. Una de sus expresiones más claras es la tendencia a reinterpretar, no sólo las experiencias molestas, sino también las experiencias normales por medio de la vía emocional. Esto se puede observar a través de la gran expansión que han adquirido las etiquetas psicológicas y los términos terapéuticos y cómo han aumentado progresivamente en los discursos sociales. Según el análisis realizado por Furedi (2004), el término síndrome "estaba totalmente 
ausente de las páginas de revistas de derecho estadounidense durante los años 1950, 1960 y 1970". Todavía en 1985 aparecía tan sólo en 86 artículos. Sin embargo, en 1990, esta cifra aumentó a 103.

Otro término significativo a este respecto es el de "autoestima" que hasta muy recientemente no ha adquirido una dimensión que refiera a problemas emocionales. Según Furedi, un estudio de Factiva sobre 300 periódicos del Reino Unido en 1980, no encontró una sola referencia a la autoestima (Figura 1). Se encontraron tres citas en 1986. En 1990, esta cifra aumentó a 103. En la década posterior, en 2000, hubo nada menos que 3.328 referencias a la autoestima. En USA se ha dado también un fenómeno paralelo. Sólo en un mes en 1993 aparecieron más de 1000 artículos en revistas y periódicos que usaban este término en Estados Unidos (Down, 1996).

En el Reino Unido y en España es igualmente creciente el uso del lenguaje terapéutico en los medios. Así pues "el infortunio" se expresa como una amenaza al bienestar personal. El rechazo, el fracaso, el sentirse sobrepasado, se consideran "riesgos para nuestra autoestima". Cuando la gente se cree vulnerable a ellos mismos o a otros se les atribuye un problema "emocional" o se asocia con el daño de cicatrices emocionales. El "emocionalismo verbal" de la cultura (Furedi, 2003) penetra en la política, el mundo del trabajo y en la vida diaria y se expande en la cultura cotidiana como medio de dar importancia a la emoción: niños de 9-10 años hablan de estrés, una palabra terapéutica, para describir un sentimiento, las guías de los Scauts también utilizan terminología terapéutica, a los alumnos de una escuela primaria de Liverpool se les ofrece cromoterapia, masajes de pies y manos o tejidos impregnados en lavanda para reducir el estrés y la agresividad. El divulgador E. Punset recomendaba en unas jornadas de innovación organizada por el gobierno autónomo andaluz y la Corporación Tecnológica de Andalucía (ENCODE), así como en su programa televisivo de divulgación científica la necesidad de educar a los niños para controlar el estrés y la ansiedad de nuevo desde pautas terapeúticas, desafortunadamente no se decía nada de cómo educarlos emocional y materialmente mediante una estrategia de empoderamiento que los habilite para hacer un ejercicio de ciudadanía activa y solidaria.

En general en los medios, pero también en las escuelas, los equipos de orientación psicopedagógicos hacen que los padres, incluso de clases populares, vayan interiorizando términos terapéuticos, que incorporan a su vocabulario cotidiano para referirse al comportamiento de los niños. De este modo, cada vez se etiquetan más a los niños con terminología psicológica como deprimidos o traumatizados. Aunque hay debate sobre la escuelafobia, a un niño enérgico o problemático, frecuentemente se le atribuye un "problema" de déficit de atención por hiperactividad. Por ejemplo, en USA, entre 1990 y 1995 se ha duplicado el número de niños diagnosticados por desorden de déficit de atención y según los expertos, por lo menos 2 millones de niños tienen este desorden. 
El vocabulario específico de los terapeutas ya no se usa sólo para problemas mentales inusuales o exóticos, términos tales como estrés, ansiedad, adicción, trauma, emociones negativas, síndromes... se usan para episodios normales de la vida cotidiana pasando a formar parte del imaginario cultural normalizado. También a nivel colectivo se usa la terminología terapéutica para describir, por ejemplo, el estado de programas de intervención urbanística, al que se alude como que es "de confianza". Para referirse a la situación después del 11 de septiembre se habla de "estado de trauma nacional", en los periódicos australianos para mostrar los efectos de una bomba terrorista en una discoteca de Bali se habla de Australia como "país en trauma"...

Está tan extendido el debate acerca de la autoestima que es fácil pasar por alto el hecho de que los problemas asociados con ella son de invención relativamente reciente (Figura 1), la explosión del término no comienza hasta mitad de los años noventa. La transformación de la autoestima en una palabra utilizada mayoritariamente refleja un patrón social más amplio que el lingüístico-cultural, que consiste en que los términos psicológicos pasan a formar parte del lenguaje de la vida cotidiana descargándolos del significado tradicional que venían teniendo. Así, actualmente, "trauma" significa poco más que la respuesta de las personas a una situación desagradable. Un estudio de Factiva en los periódicos británicos muestra un aumento espectacular en el uso de esta palabra por los periodistas en sus artículos. Lo mismo ocurre con términos como estrés o apoyo (Figuras 2 y 3 ).

FIGURA 1. El término "AUTOESTIMA" en los periódicos británicos 1980-2001 (Fuente: Estudio Factiva)

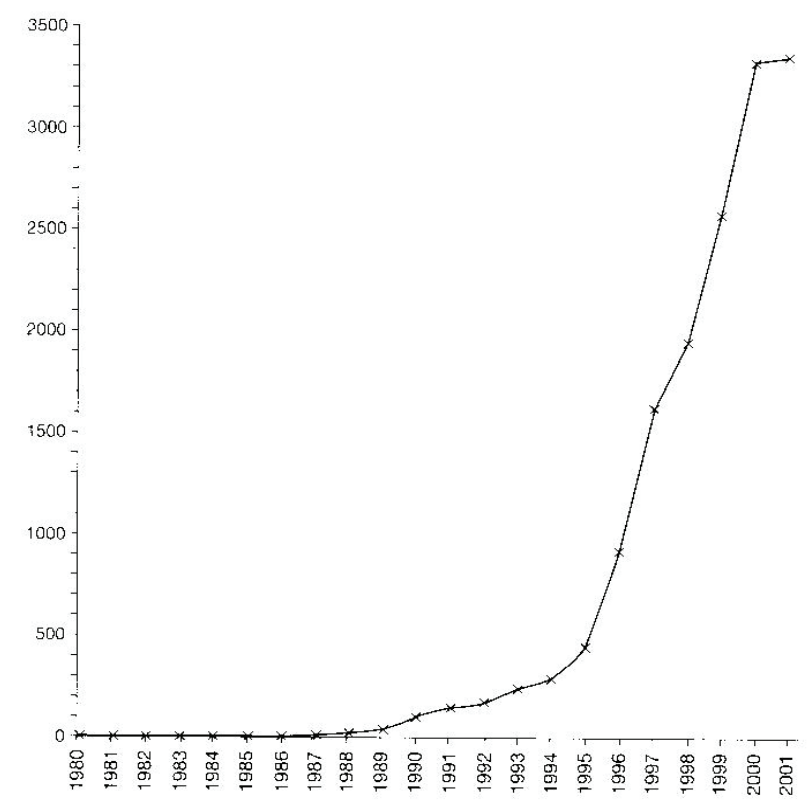




\section{FIGURA 2}

Los términos “TRAUMA” y “ESTRÉS” en los periódicos británicos 1993-2000.
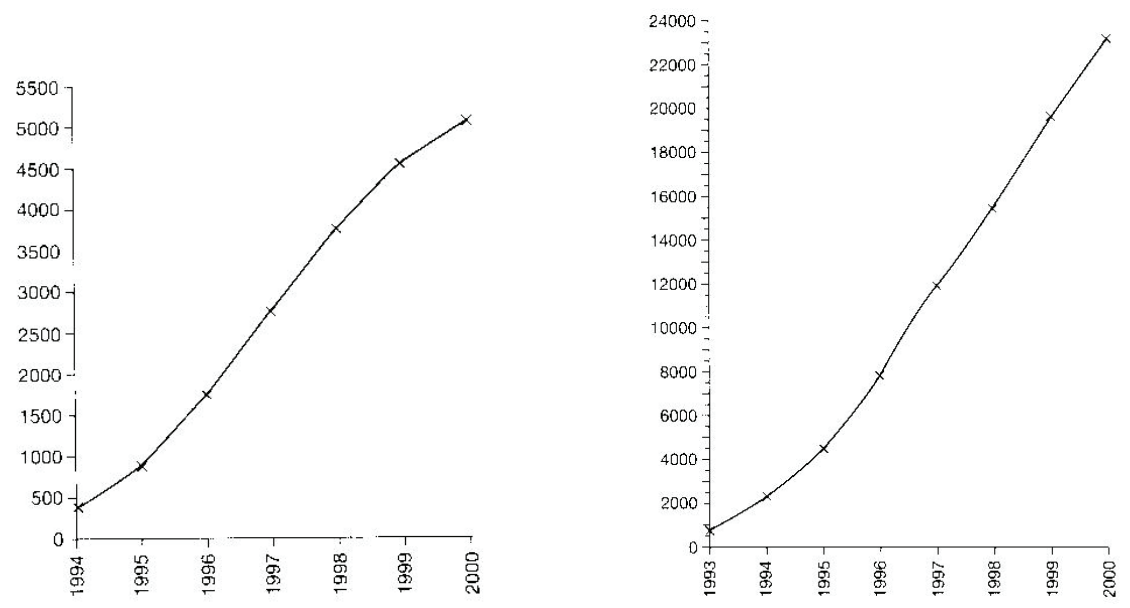

\section{FIGURA 3}

Los términos “SÍNDROME” y “APOYO” en los periódicos británicos 1993-2000.
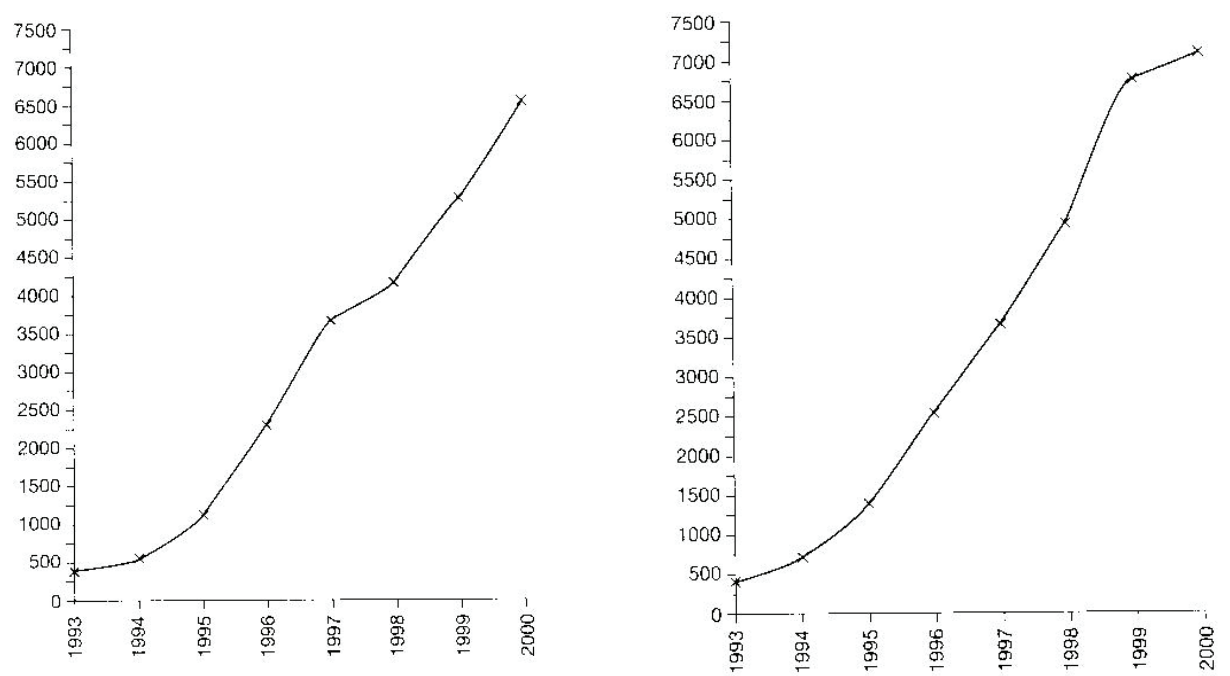

"Hoy en día, un bajo nivel de autoestima se asocia con una variedad de problemas emocionales que se dice que causan una serie de problemas sociales, que van desde la delincuencia al embarazo de adolescentes" (Furedi, 2004). La intervención cultural y artística no ha quedado fuera de esta tendencia, multiplicándose los espacios de "arteterapia" en todos los campos de las artes plásticas, musicales y escénicas. El argumento es el uso de la creatividad para desarrollar nuevas formas de comunicación para personas con baja autoestima, déficit psicofísicos, sociales o emocionales. Los hospitales se convierten en salas de exposiciones improvisadas, utilizan la música para conseguir nuevas formas de comunicación de niños debilitados por los tratamientos del cáncer, hay seminarios de grupos privados que ofrecen arteterapia para reforzar la autoestima y la conciencia personal. Las asociaciones de mujeres promueven ejercicios de Tai Chi o Qi Kung para 
mejorar dolores, ansiedad o fibromialgia. En las cárceles se hacen coros, talleres de artes pláticas, teatro... para conseguir una mejor integración y bajar los niveles de agresividad... etc. Las organizaciones que trabajan con personas con problemas de inclusión social promueven espacios vinculados con la producción artística y se crean foros y premios de pintura, teatro o fotografía dirigidos a personas vinculadas a asociaciones que trabajan con grupos de riesgo social o minorías étnicas.

Todo ello tiene mucho que ver con el aumento del "mito de la autoestima", que se usa para construir una de las características de nuestro tiempo, el "predominio actual de los sentimientos y el bienestar en la cultura como un todo". Este giro hacia el intimismo representa uno de los acontecimientos más importantes en la cultura occidental contemporánea, como señala Furedi.

Sin embargo, un análisis más cercano de la cultura terapéutica indica que no se habla tanto de la emoción, como de un problema de déficit emocional. La preocupación se manifiesta por la baja autoestima, que es siempre interpretada y entendida, según los medios, como una enfermedad invisible que socava la capacidad de las personas para controlar sus vidas. Así la creencia de que los individuos y la sociedad sufren de un déficit emocional alimenta los debates con el tema de la inteligencia emocional o la alfabetización emocional. La convicción de que las personas no pueden hacer frente emocionalmente al incremento de encuentros con personas diferentes y en distintos espacios, experiencias y relaciones, es la manera en que la cultura terapéutica entiende la condición humana.

El problema es que de ese modo la percepción del déficit emocional queda subrayada por una intensa sensación de vulnerabilidad emocional. Como resultado, la sociedad está en proceso de elaboración de una nueva definición de lo que constituye la condición humana.

Muchas experiencias que hasta ahora eran interpretadas como una parte normal de la vida han sido redefinidas como perjudiciales para las emociones de la gente. Se dice que son propensas a una variedad de condiciones y enfermedades psicológicas, como la depresión o enfermedades relacionadas con el estrés. Se generaliza en la opinión pública que muchas personas padecen de estas lesiones emocionales. Por ejemplo, como cita Furedi, en los medios ingleses con frecuencia se afirma que el número de niños que sufren de depresión va en aumento y que esto puede dar lugar a una creciente incidencia de esta enfermedad entre los adultos en el futuro. Según algunas referencias americanas, la depresión psicológica en los EE.UU, "es un castigo mayor que la pobreza". Autores como Terrence Real, sostiene que desde el comienzo del siglo XX "cada generación ha duplicado su susceptibilidad hacia la depresión". 
También cita Furedi como en Canadá parece que ha cundido esta ola de psicologismo: Según Thompson (2000), cuanto más joven es el encuestado, mayor es la prevalencia de la depresión. Apunta que este problema se debe al hecho de que los niños en Canadá han sido víctimas de más traumas infantiles en las últimas décadas que sus predecesores. Sin embargo, es difícil imaginar que Canadá se haya convertido en una sociedad más traumática en los últimos 20 años. Uno de los argumentos centrales de este libro es que lo que ha cambiado es el imaginario cultural de los traumatismos.

Así se promueve el miedo a los individuos que carecen de la capacidad de adaptación para hacer frente a los sentimientos de aislamiento, la decepción o el fracaso. Patologizando las respuestas emocionales negativas a las presiones de la vida, la cultura contemporánea, sin saberlo, anima a la gente a sentirse traumatizada y deprimida por experiencias que hasta ahora se consideraban como una rutina. El psicólogo estadounidense Martin Seligman cree que esta epidemia de la depresión se debe a la dificultad que tiene la gente para hacer frente a la decepción y el fracaso: "Embotamiento debido a la tristeza y la ansiedad justificada, creando en los niños un alto riesgo de depresión injustificada", (Furedi, 2004).

El problema es que el discurso del déficit emocional patologiza los malos sentimientos y hace que determinadas experiencias resultado de las dificultades de la vida no sean normalizadas como tales, haciendo que la supervivencia se convierta en un problema emocional. De este modo mediante esta perspectiva se va convenciendo a la gente de que se consideren a sí mismos como enfermos.

Esta forma de abordar el malestar en la cultura actual a nivel profundo lleva a convertir las incertidumbres de la vida en vulnerabilidad emocional generalizándola como una forma amplificad de riesgo. Así mediante la objetivación de la incertidumbre como riesgos fuera de control de cada uno, se cultiva una sensación de impotencia y desamparo que progresivamente va internalizándose en la conciencia mediante el discurso del déficit emocional personal.

El caso del estrés entendido como riesgo, no como resultado de las condiciones de vida es paradigmático y su aumento exponencial. En España es un discurso recurrente en los Medios referido a las situaciones de pérdida de trabajo, ritos de paso, separaciones o en la escuela y la universidad. En el Reino Unido, se afirma que los académicos universitarios se enfrentan a una epidemia de enfermedades relacionadas con el estrés y que sus estudiantes sufren cada vez más problemas de salud mental. Una encuesta indica que el 53\% de los estudiantes universitarios del Reino Unido tenía "un nivel de ansiedad patológica" y la Asociación Británica para la orientación y psicoterapia (BACP) sostiene que uno de cada diez estudiantes universitarios que buscan apoyo "ya es suicida".

Esto ha dado lugar a una enorme expansión del mercado de la terapia, pero también a 
la interiorización de las prácticas terapéuticas por parte de otros profesionales. La psicologización de la vida moderna también es evidente en el hecho de que, por ejemplo, en UK hay más terapeutas que bibliotecarios, bomberos o compañías de correo electrónico, y dos veces más terapeutas que dentistas o farmacéuticos. Significativamente, sólo la policía y los abogados son más numerosos, pero sólo en una proporción de menos de dos a uno en ambos casos. La asimilación de la ética y las prácticas terapéuticas por la mayoría de instituciones y profesiones tales como profesores, gestores culturales, abogados y trabajadores sociales ha sido bien documentada. "La gestión de la subjetividad se ha convertido en una tarea central para la organización moderna”, señala Nikolas Rose (1990), en su estudio exhaustivo de la evolución de la institucionalización de la cultura terapéutica en Gran Bretaña.

\section{CONSECUENCIAS SOCIOCULTURALES Y POLÍTICAS DE LA CULTURA TERAPÉUTICA: EL PAPEL DEL GESTOR CULTURAL.}

Todo lo anterior remite a un cambio importante en el papel que se asigna a la condición humana y a las políticas públicas que venían construyendo el modelo de bienestar. En este contexto, el papel del Estado se traslada también al control de las políticas de lo íntimo, directamente, a través de la promoción de espacios de asesoramiento terapéutico financiado desde el erario público vinculado a las políticas culturales, sociales, judiciales... y a nivel indirecto, propiciando espacios de comunicación en los Medios en los que se refuerza esta opción de intervención en lo íntimo por parte de la sociedad y el discurso experto psicologizado.

De este modo se hace efectiva, como señala Furedi, la convicción de que las desigualdades sociales son experienciadas a través de "mecanismos psicológicos" que vinculan la pobreza a la provisión de salud individual, promoviendo políticas y técnicos terapéuticos. A partir de ahora "emociones, salud y justicia distributiva están por ello intimamente relacionadas en el mundo occidental". Así aparece cada vez más frecuentemente el concepto de "estar bien", derivado de un conjunto de principios alrededor de los cuales podría organizarse una nueva visión del "bienestar" positivo. Para Hoggett (2000) y sus correligionarios creyentes en ese modelo, el "Estar bien" se define esencialmente en términos de salud mental. La cuestión es que estos autores que defienden la necesidad de una gobernaza terapéutica, la consideran como algo inocuo e incluso potencialmente empoderante, lo que conlleva una cultura política que tiene en realidad poca confianza en la capacidad individual y colectiva de los ciudadanos para actuar de forma responsable sin la ayuda de profesionales pagados, a los que se les supone que son los que mejor conocen sus intereses, como si ellos estuvieran fuera del campo ideológico.

Sin embargo, esto tiene una carga política importante, pues la transformación de los ciudadanos en pacientes o clientes, implica potencialmente alterar las relaciones entre las personas y las instituciones públicas. Esto supone, como señala V. Pupavac 
(2001), la erosión del concepto de contrato social de los ciudadanos como sujetos racionales autónomos. El nuevo contrato social terapéutico reafirma el presupuesto paternalista de que el sujeto vulnerable emocionalmente necesita de la gestión y el apoyo de la administración pública.

En este contexto, el papel del gestor cultural se ve limitado a paliar la creciente vulnerabilidad social. Sin embargo, no tiene que ser necesariamente así, pues si bien se puede asumir y potenciar esta lógica de subordinación a la vulnerabilidad emocional, muy funcional a los intereses de los grupos dominantes, que implica también desmovilización social, atemperando las emociones de miedo, ansiedad o agresividad, también es posible utilizar los resortes de la cultura colectiva para promover desde la cultura estrategias de resistencia. Es posible canalizar a través de las prácticas culturales estas pulsiones, de forma que sirvan para descubrir y enfrentar las causas que originan el miedo, la frustración o la ansiedad y posicionarse como ciudadanos en espacios que no tienen por qué acentuar las dimensiones de riesgo o ser traumatizantes, sino potenciadoras de las pulsiones de vida a través del descubrimiento de las potencialidades de lo colectivo, el divertimento, rescatando el sentido y la alegría de la vida que a pesar de las supuestas amenazas, subsiste en la condición humana y se manifiesta mediante la cultura.

Si hace 30 o 40.000 años la inteligencia social, la capacidad de imitar y la inteligencia natural han sido una condición necesaria para que apareciera la cultura en el homínido (Winfel, 2009; Mithen, 2009), es necesario volver a confiar en la capacidad humana para hacer un buen uso de esa inteligencia. Al destruir $u$ olvidar esos principios, nos vamos diluyendo como especie que ha alcanzado un cierto grado de autonomía y capacidad autorganizativa, perdemos humanidad, para convertirnos en meros seres asustados, vulnerables y dependientes de lo que dicta el grupo "experto" sobre qué debemos sentir y creer. Ese grupo experto, ahora cómodamente instalados en la cultura terapéutica desde el Estado o el mercado, que como nuevos sacerdotes cancerberos de la decencia emocional, tratan de controlar desde su ciencia el ethos, como si no estuvieran sometidos a la dialéctica de poder del mundo y sus luchas.

Teniendo en cuenta que el modelo actual de glocalización ha posibilitado una enorme concentración del poder económico-financiero y que la salud se ha convertido en un ámbito de inversión para las grandes compañías con sus lobbies de poder que penetran en las instituciones públicas y privadas, esta cultura, que convierte al ciudadano en paciente, es decir, sufridor pasivo de los intereses de aquellos que pueden ser sujetos e imponer las reglas de juego, implica un empobrecimiento muy problemático de los logros civilizatorios de la modernidad y de los derechos de ciudadanía que han venido conformando el contrato social moderno.

Como apuntan diferentes autores, lo que ahora se trata de controlar desde los nuevos dispositivos terapéuticos es la disidencia. Esta apuesta, como dice Furedi, implica en 
realidadunaintoleranciahacialas emociones disidentes: "Desdeinstitucionesyprofesionales dedicados a gestionar cómo debe sentir la gente, se están impulsando sistemáticamente ciertas actitudes emocionales y reprimiendo otras". La sociedad contemporánea necesita certezas acerca de sus creencias y tiene dificultades para transmitir una visión clara de qué es un mundo justo, pero tampoco en este modelo parece que se ofrezca a la gente un universo claro de sentido. Precisamente, la confusión entre proveer sentido y aplicar terapias desde las políticas es lo que otorga una considerable oportunidad de expandir el espacio de influencia de las elites. Hoy los grupos dominantes en el panorama cultural necesitan ganar confianza para decir a la gente en qué creer, pero resulta bastante más cómodo, instruir a la gente sobre qué y cómo sentir.

$\mathrm{Al}$ instruir sobre qué tiene que sentir la gente, se refuerza la estrategia de generar conformidad con valores terapéuticos claves a través de la gestión de la subjetividad. Este es un espacio compartido por los profesionales de la cultura y el arte, además de los expertos psicólogos. Pero ¿Quién decide cuál es el camino positivo para gestionar estos sentimientos? ¿Los niños, los padres, los profesores, los gestores, los psicólogos, los técnicos, los políticos...? ¿Qué derecho tienen las instituciones para imponer su versión de lo que es la gestión positiva de los sentimientos?

Para responder a estas cuestiones hay que considerar y separar la dimensión educadora de la cultura que capacita a las personas para el ejercicio de la ciudadanía y controlar su propia vida, de la otra vía que supone instruir y dictarles normas que no responden necesariamente a sus intereses. En el mundo actual hay bastantes razones para el enfado y las emociones negativas. La represión o la sublimación de las emociones sin ir al fondo de las causas del malestar no resuelve los problemas, simplemente actúa como control de los conflictos. Instruyendo a la gente para que asuman sus sentimientos de forma individual se promueve la aquiescencia y el conformismo. Así al considerar las emociones llamadas negativas como una enfermedad, se distrae la atención del hecho de que posiblemente las condiciones que las motivan son las que necesitan ser curadas (Summerfield, 1996).

La creencia en que el estado emocional del individuo determina lo que sucede en la sociedad, lleva a un determinismo emocional que condiciona la manera en que los problemas sociales son percibidos. Este determinismo es particularmente poderoso en lo que respecta a la percepción actual de la infancia y la juventud. Según este nuevo dogma, los problemas experimentados y causados por los adultos están enraizados en el mundo emocional de los niños y los jóvenes. Por ello las instituciones públicas piensan que tiene la obligación de proteger y modificar los sentimientos de los niños y jóvenes, hablando de alfabetización emocional. También en el empleo y las situaciones de crisis se incluye un experto en emociones para resolver los problemas en el Reino Unido y USA, que en caso de no ser aceptado puede tener consecuencias sancionadoras (Furedi, 2004). 
Este nuevo control de la corrección emocional afecta especialmente a la esfera del arte. En USA y UK la libertad de expresión ha sido puesta en cuestión por aquellos que argumentan que, dado que las palabras pueden herir y causar daños psicológicos, las supuestas víctimas deberían ser protegidas de este riesgo. Así en este contexto incluso el arte queda bajo sospecha. Los artistas se enfrentan a la censura y se ven obligados a cerrar exposiciones bajo el pretexto de que sus pinturas ofenden a determinado público. Parece ser que el clima actual de corrección emocional no puede tolerar el derecho a ser incorrecto. Actos, exposiciones o palabras son frecuentemente denunciadas por causar daño u ofensa. La afirmación "me siento ofendido" implica una demanda de censura, lo que se ve favorecido por el mercado de los gabinetes de abogados. Rauch (1993) afirma que los ataques actuales al libre pensamiento que considera que la gente debe ser sancionada por sostener pensamientos políticamente incorrectos conduce inexorablemente "hacia la Inquisición”. Este autor es particularmente crítico con la noción cada vez más expandida de que "las palabras e ideas hirientes son una forma de violencia" que conducen a "la criminalización de la crítica". Críticos como Rauch son frecuentemente acusados de olvidarse de las víctimas de estas palabras, mientras que desde los regulacionistas de los sentimientos se demanda aún más reglas y regulaciones de la conducta.

Mientras tanto, la indecencia cotidiana de la pobreza, la explotación y la expoliación de los recursos de las mayorías vulnerabilizadas queda invisibilizada. Mientras la sociedad se judicializa para aquellos con poder para pagar sus causas, se individualiza restringiendo los ámbitos de sociabilidad, apoyo mutuo y afectividad (sentirse afectado por el otro amorosamente).

El crecimiento del consenso elitista contra la libre expresión desde lo políticamente correcto, ejemplifica cómo la politización de las emociones tiene perturbadoras consecuencias autoritarias, tales como la marginalización de las ideas no conformistas. Esto es problemático, teniendo en cuenta que a través de la historia cualquier idea nueva ha causado estupor y ofensa en determinados grupos sociales, desde el sufragio universal al trasplante de órganos, la contracepción o la legalización del divorcio, fueron en su momento cuestiones ofensivas para los estándares de la "decencia pública". Cuando cuestiones de subjetividad privada pasan al control de las instituciones públicas y de la justicia, como dice Furedi, la terapia y la justicia se confunden, se llega a la erosión de los derechos y a la arbitrariedad en su ejercicio, lo que da lugar a un nuevo conservadurismo basado en los sistemas de control basados en el cultivo del sentimiento de vulnerabilidad, indefensión y dependencia de las personas.

La transformación de la enfermedad en una identidad es potencialmente un problema muy serio para la salud pública. Una de las incidencias más claras está en el modo en que se ha contrapuesto la relación entre experiencia individual y social. El sentido pasivo que tiene la construcción actual de la salud individual convierte a la mayoría de ciudadanos 
en seres vulnerables y en riesgo, lo que hace que no se asuman los desafíos y riesgos que siempre supone la vida. En esta nueva cultura de la terapia se instruye más para identificar problemas que para trascenderlos y buscar respuestas.

Ante esta situación no dejan de proliferar nuevos espacios culturales alternativos y movimientos sociales que tratan de dar nuevas respuestas a los riesgos, propiciando ámbitos de reconstrucción del conocimiento para la salud desde la experiencia de grupos sociales y de la ciudadanía con formatos no terapéuticos. Estos grupos se expresan a través de las redes que propician los nuevos formatos de comunicación digital y a través de espacios en los que se trata de recuperar la inteligencia natural ${ }^{3}$ y social que promueve nuevas formas de convivencia sanas.

Algunas fórmulas societarias rescatadas y actualizadas de la cultura tradicional se renuevan como en el caso de los inmigrantes chinos, las nuevas formas de cooperación on-line entre otras recogidas por R. Sennett (2012) en Together como medidas de resistencia de los vulnerabilizados.

Movimientos sociales como el que promueve la vida lenta (Slow, Honoré, 2005) o la comida pausada (Slow Food, Petrini, 2006) mediante la reconstrucción de nuevos espacios de aprendizaje en convivencia en torno a una nueva forma de gestionar la vida, el consumo (Gavira y González, 2010), la música, el arte o el alimento a través de la convivencialidad que ya Illich señalaba como parte de la condición humana. El ciberpunk o el movimiento por el software libre (Mas Hernández, 2005) son otros casos de resistencia independiente a la subordinación emocional de la cultura terapéutica.

En el campo de la cultura sanitaria también surgen espacios alternativos que promueven el uso de la cultura y la experiencia desde propuestas más integrales, seguras y autónomas, enfrentando las lógicas de instrucción dominantes de todos los espacios de la vida.

También proliferan los movimientos artístico y culturales que dan lugar a experiencias creativas que orientan al empoderamiento de los sujetos reforzando sus capacidades para trascender los problemas a través del teatro, producciones multimedia o apuestas como las del Museo de la Precariedad de Albinet, una propuesta artística de Patriçia Le Nenan y Jean-Françoise Isaïa, en la que a través del arte se reconstruye y refuerza la confianza colectiva de las personas excluidas de un barrio urbano y de sus potencialidades colectivas. Otros ejemplos nacen de otras maneras de entender la cultura desde la creación y reconocimiento intercultural como "transacciones / fadaiat", producido por Carta de Ajuste/BNV Producciones o Carta Blanca a partir del Espacio Arteypensamiento vinculado a la Universidad.

3. Se entiende natural en el sentido biofísico de relación con el resto de la naturaleza. 
Por último el movimiento de los indignados ha mostrado cómo autorganizarse y empoderarse huyendo de los expertos, construyendo cultura y conocimiento de forma horizontal, sin caer en los viejos formatos de los movimientos sociales clásicos que reducían todo a la acción sin considerar las implicaciones afectivas, lo que ha hecho escribir al profesor Víctor Sampedro:

"No se puede parar un movimiento que propone una transición permanente protagonizada por la sociedad civil, frente a la transacción pactada desde la derrota. La capitulación como punto de partida y de llegada. La victoria de los mismos de siempre.

No pararán el $15 M$ porque no habrá trincheras en el espacio público, sino espacios liberados frente al búnker. Las sentadas, no las barricadas, aren el camino. Porque los cuerpos, no los adoquines, se abrazan frente a la amenaza de los uniformados. Les miran, interpelan... exigen respuesta. Se alzan y abren nuevas sendas." En «\#12M15M: No lo pararán. Bruce Lee vs. Charles Bronson» Diario Público.es, 13 de Mayo de 2012.

Todos estos son ejemplos ilustrativos de formas de gestionar la salud social por parte de ciudadanos, artistas y profesionales de la cultura que el gestor cultural no puede dejar de considerar. Especialmente, teniendo en cuenta la ecorresponsabilidad que ha de asumir como sujeto mediador con los grupos e instituciones con los que interactúa. 


\section{REFERENCIAS BIBLIOGRÁFICAS}

Bauman, Zygmunt (2001) La sociedad individualizada. Madrid: Cátedra

Beck, Ulrich (2001) ¿Qué es la globalización? Falacias del globalismo, respuestas a la globalización. Barcelona: Paidós.

Boltanski, Luc y Chiapello, Eve (2002) El nuevo espíritu del capitalismo. Barcelona: Akal.

Castells, Manuel (1995) La ciudad informacional. Tecnologías de la información, estructuración económica y el proceso urbano-regional. Madrid: Alianza Editorial.

Castel, Robert (2004) Las trampas de la exclusión. Tropía.

Downs, Donald (1996) More than Victims: Battered Women: The Sindrome Society, and the Law. Chicago: University of Chicago Press.

Gavira, Lina y González, Francisco (2010) "Redes alimentarias como resistencia al modelo de globalización dominante: El caso de Slow Food." en Patrimonio cultural en la nueva ruralidad andaluza. PH Cuaderno de pensamiento, $\mathrm{n}^{\circ}$ 26. Instituto Andaluz del Patrimonio Histórico.

Gavira, Lina (1995) "New Labour-Related Poverty and Deprivation in the Current Situation: Andalusia and Scotland". RUSSTIC Seminar. Human Capital and Mobility Program.

Gavira, Lina (2002) Andalucía sobreviviendo a la glocalización. Sevilla: Mergablum.

Guattari, Félix y Rolnik, Suely (2006) Micropolítica. Cartografía del deseo. Madrid: Traficantes de Sueños.

Hoggett, Paul (2000) en Lewis et alter, Social Policy and the Emotions. Macmillan Press. Honoré, Carl (2005) Elogio de la lentitud. Un movimiento mundial desafía el culto a la velocidad. RBA.

Mas i Hernàndez, Jordi. (2005) Software libre: técnicamente viable, económicamente sostenible, socialmente justo. http://www.infonomia.com.

Mithen, Steven (2009) "Punset entrevista a Alan Winfield que emplea robots para el estudio del origen y evolución de la cultura" http://www.phpia.net/content/punsetentrevista-alan-winfield-que-emplea-robots-para-el-estudio-del-origen-y-evolucionde-

Petrini, Carlo (2007) Bueno, limpio, justo. Ediciones Polifemo.

Pupavac, Vanessa (2001) “Therapautic governance: psicosocial interventionand trauma risk management” cit. por Furedi, 2004.

Rauch, Jonathan (1993) Kind Inquisitors: The New Attacks on Free Thoughts. Chicago: The University of Chicago Press. 
Roberston, Roland (1992) Golbalization: Social Theory and Global Culture. Sage.

Rose, Nikolas (1990) Governing the Soul: The Shaping of the Private self. Routledge.

Sennett, Richard (2012) Together. The Rituals, Pleasures and Politics of Cooperation. Penguin Books.

Sennett, Richard (2000) La corrosión del carácter: las consecuencias personales del trabajo en el nuevo capitalismo. Barcelona: Anagrama.

Summerfield, Derek (1996) The Impact of War and Atrocity on Civilian Populations: Basic Principles for NGO Interventions and a Critique of Psychosocial Trauma Projects. ODI.

Thompson, Gregory (2000) Study warms about rise in childhood trauma cases. Alberta: University of Alberta. www.alberta.ca

Winfield, Alan (2009) "Punset entrevista a Alan Winfield que emplea robots para el estudio del origen y evolución de la cultura" http://www.phpia.net/content/punsetentrevista-alan-winfield-que-emplea-robots-para-el-estudio-del-origen-y-evolucionde- 